\title{
Laser Beam Micro Welding With High Brilliant Fiber Lasers
}

\author{
Felix SCHMITT, Benjamin MEHLMANN, Jens GEDICKE, \\ Alexander OLOWINSKY, Arnold GILLNER, Reinhart POPRAWE \\ Fraunhofer Institute for Laser Technology, Steinbachstr. 15, 52074 Aachen, Germany \\ E-mail: felix.schmitt@ilt.fraunhofer.de
}

\begin{abstract}
Spatial power modulation for laser beam micro welding by superposing an oscillation movement to the feed direction is gaining more and more importance, especially for welding of difficultto-weld materials, e.g. aluminum, copper or zinc-coated steel sheets. Up to now, there are no adequate machine techniques available providing oscillation frequencies of more than $5 \mathrm{kHz}$. Examinations about the dynamic behavior of conventional galvanometric scanners with integrated oscillation movement ("wobble function”) are presented. These systems are commonly used for laser beam micro welding but their dynamic behavior at high oscillation frequencies is not known yet. A focus of the examinations is on alternative scanning systems, presenting their characteristics and their applicability for laser beam micro welding. Applications presented are welding experiments for an adapted seam width in bead-on-plate welding.

DOI:10.2961/jlmn.2010.03.0003
\end{abstract}

Keywords: micro welding, galvanometer scanner, spatial power modulation, beam deflection

\section{Introduction}

High brilliance lasers such as fiber laser provide a high focusability at high average laser power. Following advantages can be counted for laser beam micro welding:

- Small spot diameter enables welding of thin metal sheets down to $25 \mu \mathrm{m}$ thickness

- High intensity is leading to good incoupling of laser beam into the workpiece

- Smaller required aperture leads to an increase in beam deflection scanning frequencies

The small spot diameter enables welding of thin metal sheets. In Fig. 1, left, a top-view of a welding seam in a thin metal foil with a thickness of $25 \mu \mathrm{m}$ is given. At welding speeds of $833 \mathrm{~mm} / \mathrm{s}$ a very smooth and homogeneous welding seam is generated [1]. However, a small spot size is leading to a narrow welding seam which is not preferred for overlap welding due to limited joint strength. In Fig. 1, right, a cross-section of an overlap joint in stainless steel is shown. Due to the good beam quality of the laser source a welding geometry with a high aspect ratio is generated.
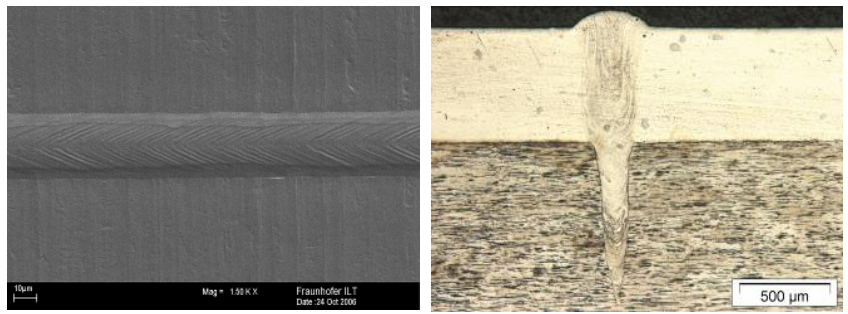

Fig. 1 Laser beam micro welding of thin foils (left) and an overlap joint with high aspect ratio (right)

A wider welding seam can be achieved by an oscillation movement superposed to the feed direction. With galvanometric scanners such an oscillation movement is possible but these scanners are limited in their dynamic behavior. With increasing welding speed, oscillation frequencies of more than $5 \mathrm{kHz}$ are necessary. The use of high dynamic oscillation techniques leads on the one hand to a stabilization of the welding process, e.g. in terms of welding depth and melt pool behavior [2], on the other hand to an increase of process velocity, e.g. for polymer welding [3]. The applied temporal and spatial power modulation in laser beam micro welding stabilizes the welding process, which is important for materials that are difficult to weld, e.g. copper or aluminum. Due to the enlargement of the weld pool geometry when applying spatial power modulation the circulation speed around the keyhole is reduced and potential process errors like humping or spattering are prevented.

\section{Temporal and spatial power modulation for laser beam micro welding}

Temporal power modulation is the variation of the laser power during the laser pulse without considering a possible relative movement between laser beam and workpiece. Pulse forming enables adjustment of the laser power emissions to the different absorption conditions of the material in solid and liquid phase. In contrast to a square pulse, a continuous inclining ramp of laser power output is realized at the beginning of the pulse via pulse forming. This leads to a reduction of splatter at the beginning of the process. A continuous reduction of the laser power output at the end of the pulse reduces undercut [4]. A prepulse phase consisting of a low output power plateau supports stabilization of welding process by preheating the work area when applying spot welding [5].

Especially when welding highly reflective materials pulses with high peak power at the beginning of the pulse are used. Furthermore, superposition of a metallurgical pulsing which is a high frequency modulation ( $\mathrm{f}>5 \mathrm{kHz}$ ) reduces the weld pool dynamics. This superposition of a high frequency modulation also improves the seam surface [5]. Furthermore, pulse forming enables the control of the undercooling of the melt pool and with it the solidification of the melt. This leads to a high nucleation rate resulting in a fine-grained microstructure. Welding of Titanium (Ti 
Grade 1) using pulse forming has shown a refinement of the grain microstructure by $28 \%$ [6].

Spatial power modulation refers to a relative movement between laser beam and workpiece by superposing an oscillation movement to the feed direction which forms the welding geometry. Generally, the superposed movement can vary in its geometry, e.g. a linear movement in feed direction or perpendicular to that, a circular geometry or any Lissajous figure. The impact of these oscillations on welding of metals depends on the used oscillation frequency. According to [2] there are three types of interaction possible:

- Influencing the keyhole geometry (interface gaseous-liquid)

- Influencing the weld pool geometry (interface liquid-solid)

- Influencing the temperature gradient in the weld pool

The time scale of the interface gas-liquid is in the range of a few $10 \mu \mathrm{s}$ and of the interface liquid-solid is between $100-1000 \mu$ s. Conventional galvanometer scanners are capable of oscillation frequencies in the lower kilohertz range what only influences the weld pool geometry. In order to influence the keyhole geometry oscillation frequencies of $100 \mathrm{kHz}$ are required [2]. Using a high overlap by applying an enlarged amplitude reduces significantly the weld pool dilution of an aluminum-copper overlap weld. Because of the available system technology the oscillation frequency is limited to $2 \mathrm{kHz}$ [2]. An improvement of the seam quality is reported for an overlap weld of zinc-coated steel sheets by spatial power modulation [7]. The process speed is again limited by the available system technology. The laser beam welding of aluminum produces a homogeneous weld bead and a reduction of the amount of pores is detected according to [8]. The laser beam is oscillated linear in feed direction by using a single galvanometric mirror with a maximum frequency of $1500 \mathrm{~Hz}$. This leads to an extension of the weld pool and is similar to the twin-spot technique used for laser beam welding of aluminum and zinc-coated steel sheets [9]/[10].

The resulting paths of superposing a circular oscillation with the feed are shown in Fig. 2 for two different feed rates (left: $50 \mathrm{~mm} \cdot \mathrm{s}^{-1}$; right: $200 \mathrm{~mm} \cdot \mathrm{s}^{-1}$ ) at an oscillation frequency of $1000 \mathrm{~Hz}$ and an amplitude of $0.1 \mathrm{~mm}$. The dimension of the laser beam is not considered. The feed direction corresponds to the $\mathrm{x}$-axis. The correlation between oscillation amplitude $a$, feed rate $v$ and oscillation frequency $f$ has to be considered for the appropriate parameters. The vectorial relation is shown in equation (1)

$$
\left(\begin{array}{l}
x \\
y
\end{array}\right)=\left(\begin{array}{c}
-a \cdot \cos (2 \cdot \pi \cdot f \cdot t)+v \cdot t+a \\
-a \cdot \sin (2 \cdot \pi \cdot f \cdot t)
\end{array}\right)
$$

where $a$ is the radius of the circles covered by the laser beam at feed rate $v$ of $0 \mathrm{~mm} \cdot \mathrm{s}^{-1}$ and at a set frequency. With increased feed rate circles are deformed to a spiral. If feed rate is being increased the oscillation frequency has to be increased as well, otherwise no overlap of the circles will take place (Fig. 2, right). This correlation reveals that merely high dynamic scanner systems enabling oscillation frequencies in multi-kHz-range lead to a further increase of process velocity.
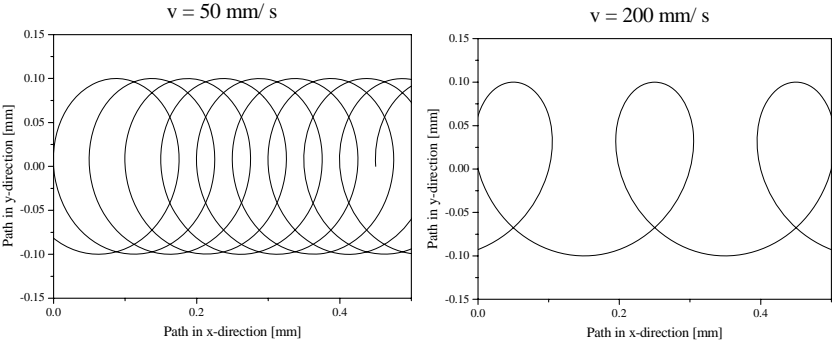

Fig. 2 Oscillation function dependent on feed rate in feed direction (left: $50 \mathrm{~mm} \cdot \mathrm{s}^{-1}$, right: $200 \mathrm{~mm} \cdot \mathrm{s}^{-1}$ )

Increasing the feed rate leads to an insufficient overlap of the single loops. To characterize the process a definition for the weld overlap is set analogous to the classical single spot welding. As a first step the beam path is taken into account without the consideration of the laser beam diameter. Assuming small feed rates and well shaped circles a simplification is valid considering only the intersections of the path with the $\mathrm{x}$-axis $(\mathrm{y}=0)$. If increasing the feed rate this simplification gets inaccurate. For an exact description of the overlap the extrema are examined $(d x / d t=0)$. The overlap $\mathrm{n}$ is defined in equation (2). $x_{\max }$ and $x_{\min }$ are the local maxima and minima of the movement in feed direction. These extrema are dependent on radius of the circular movement $a$, feed rate $v$ and oscillation frequency $f$, resulting in a dependency of $n$ as well. The geometric meaning of this definition is shown in Fig. 3.

$$
\begin{aligned}
n= & \frac{X_{\max }(\text { period } 1)-X_{\min }(\text { period } 2)}{X_{\max }(\text { period } 1)-X_{\min }(\text { period } 1)} \cdot 100 \% \\
n= & \frac{2 \cdot a \cdot \sqrt{1-\left(\frac{v}{2 \pi \cdot f \cdot a}\right)^{2}}-v \cdot\left(3 \pi-2 \cdot \arcsin \left(\frac{v}{2 \pi \cdot f \cdot a}\right)\right)}{2 \cdot a \cdot \sqrt{1-\left(\frac{v}{2 \pi \cdot f \cdot a}\right)^{2}}-v \cdot\left(\pi-2 \cdot \arcsin \left(\frac{v}{2 \pi \cdot f \cdot a}\right)\right)} \cdot 100 \%
\end{aligned}
$$

The left figure displays the movement on the $\mathrm{x}$-axis over time. Local minima and maxima are plotted and the resulting distances that are used in the overlap definition are displayed. The right figure shows the laser path in $\mathrm{x}$ and $y$-direction and is displaying the calculated local extrema of $\mathrm{x}$-direction. The defined overlap compares the distance between a loop to the following one and the loop diameter.
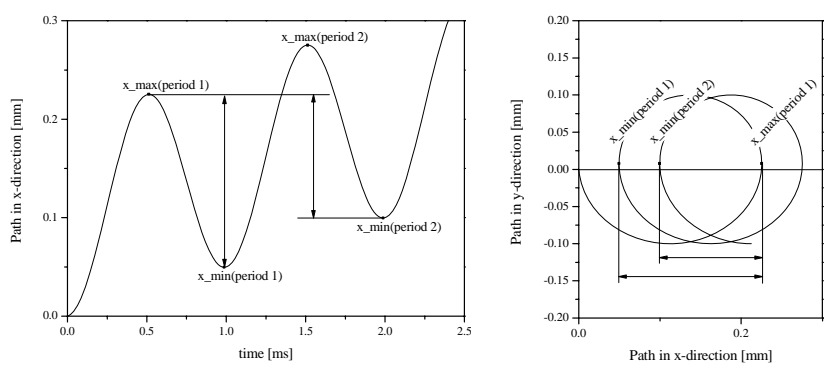

Fig. 3 Graphical definition of the overlap $n\left(v=50 \mathrm{~mm} \cdot \mathrm{s}^{-1}\right.$, $\mathrm{a}=0.1 \mathrm{~mm}, \mathrm{f}=1 \mathrm{kHz}$ ); left: path in feed direction over time displaying the local extrema; right: path in $\mathrm{x}$ - and $\mathrm{y}$ direction showing the same local extrema as in left fig.

The paths displayed in Fig. 2 exhibit an overlap of $71.5 \%$ for $50 \mathrm{~mm} \cdot \mathrm{s}^{-1}$ and an overlap of $-81 \%$ for $200 \mathrm{~mm} \cdot \mathrm{s}^{-1}$ which has no overlap at all. A homogeneous 
weld seam can be created when using a high overlap. The potential of a superposed oscillation movement at laser beam welding has been demonstrated in the given literature; however the dynamic of the welding processes is limited through the insufficient system technology. The overlap as a function of frequency and amplitude is displayed in Fig. 4 for two different feed rates. In order to realize the required overlap the oscillation frequency must be increased for smaller amplitudes and for higher feed rates.
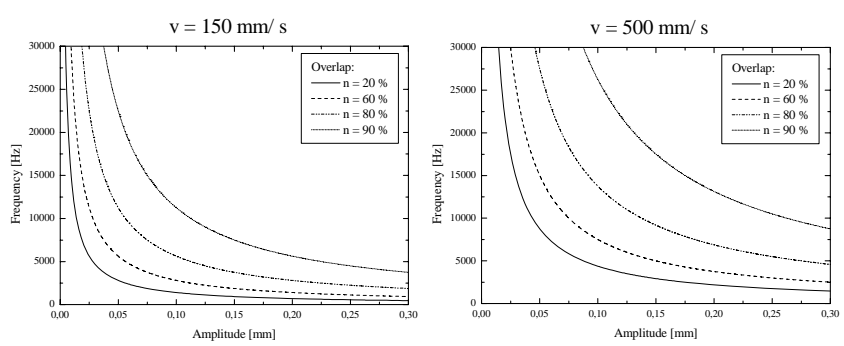

Fig. 4 Overlap as a function of oscillation frequency and amplitude at two feed rates (left: $\mathrm{v}=150 \mathrm{~mm} \cdot \mathrm{s}^{-1}$, right: $\mathrm{v}=$ $500 \mathrm{~mm} \cdot \mathrm{s}^{-1}$ )

Technically desired parameters for laser beam micro welding of thin metal foils are oscillation amplitude between $0.05 \mathrm{~mm}$ and $0.1 \mathrm{~mm}$ and a feed rate greater than $150 \mathrm{~mm} \cdot \mathrm{s}^{-1}$. This concludes that a system technology enabling oscillation frequencies greater than $20 \mathrm{kHz}$ would be needed to apply overlap greater than $80 \%$.

\section{Current system technology}

Galvanometric scanners are currently used as fast, flexible beam deflectors. These systems are able to superpose the feed movement with a circular oscillation of the laser beam. Using a Hamamatsu position sensitive detector (PSD S2044, measuring field $4 \times 4 \mathrm{~mm}^{2}$, rise time $0.3 \mu \mathrm{s}$, position resolution $0.6 \mu \mathrm{m}$ ) two galvanometric scanners are analyzed for their dynamic characteristics. The scanners are a Scanlab SK $1020^{\circledR}$ with an optical aperture of $20 \mathrm{~mm}$ and a Scanlab Scancube $7^{\circledR}$ with an optical aperture of $7 \mathrm{~mm}$. Both scanners exhibit a growing deviation from the preset amplitude if the oscillation frequency is increased (Fig. 5).
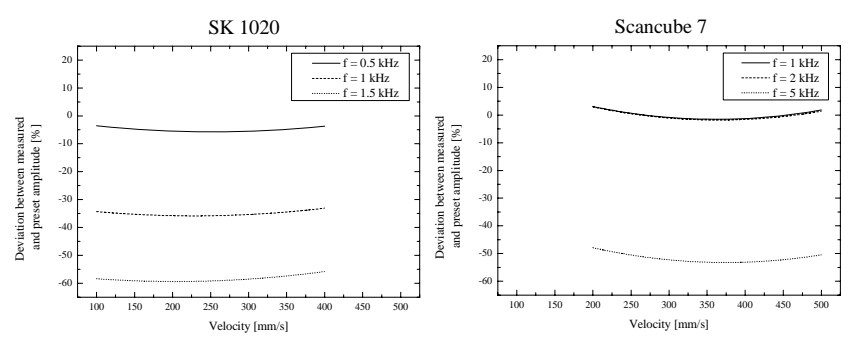

Fig. 5 Deviation between measured and preset amplitude for two different galvanometer scanners dependent on feed rate and oscillation frequency (left: SK 1020, right: Scancube 7)

The preset oscillation amplitude is $0.1 \mathrm{~mm}$. The graphs show the deviation of the measured amplitude to the preset amplitude over the feed rate and oscillation frequency. The amplitude of the larger scanning mirror (SK $1020^{\circledR}$ ) deviates from the preset amplitude about $5 \%$ at a frequency of $0.5 \mathrm{kHz}$. The measured deviation for a feed rate between
$100 \mathrm{~mm} \cdot \mathrm{s}^{-1}$ to $400 \mathrm{~mm} \cdot \mathrm{s}^{-1}$ is almost constant. An increase of the oscillation frequency decreases the amplitude. A reduction of $35 \%$ occurs at $1 \mathrm{kHz}$ and a reduction of $60 \%$ at $1.5 \mathrm{kHz}$. Since the mirrors of the Scancube $7^{\circledR}$ are smaller and newer system technology is used, a reduction of the amplitude does not occur for $1 \mathrm{kHz}$ and for $2 \mathrm{kHz}$. At an oscillation frequency of $5 \mathrm{kHz}$ the decrease of the amplitude compared to the preset one is $50 \%$.

In order to overcome these dynamic limitations of galvanometric scanners a new scanner system is needed. The required specifications are:

- Highly dynamical oscillation ( $\mathrm{f}>20 \mathrm{kHz}$ )

- Geometric flexibility (Lissajous figures: e.g. circle, line, ellipse)

- Designed for laser power up to $100 \mathrm{~W}(\mathrm{cw})$

- Small overall size with optical aperture $>3 \mathrm{~mm}$

- Deflection angle $<3$ mrad (@ f = 80 mm, telecentric)

A qualitative comparison of different beam deflection systems is given in Table 1. Criteria of the comparison are the size of the deflection system regarding their integrability in superordinated moving systems, the geometric flexibility of the executed contours (line, circle, ellipse, Lissajous-figures), the number of optical elements to create a 2D oscillation geometry and the dynamic behavior.

Electro-optical deflectors and polygon scanner are not taken into account. Electro-optical deflectors are excluded due to their characteristic setup (beam guiding in a crystal over $150 \mathrm{~mm}$ with apertures $<3 \mathrm{~mm}$ ), their unknown damage threshold at laser powers around $100 \mathrm{~W}(\mathrm{cw})$ and their high costs. Polygon scanners are eliminated because of their limited dynamic behavior and limited geometric flexibility.

Mechanical scanners are rotational devices. Here, a reflective wedge blank is mounted on a conventional high speed spindle. Rotational frequencies of up to $16 \mathrm{kHz}$ are possible but these systems are very sensitive against unbalanced masses what is mandatory because of the geometry of the mirror. Due to the rotation of the element exclusively circle-shaped and ellipse-shaped geometries are possible.

Galvanometric scanners have a high potential for this application but these systems are limited regarding their dynamic behavior what has been explained above. Due to their geometric flexibility and combination of oscillation and feed direction in one device they are up to now the appropriate choice.

Piezo actuators are available as $1 \mathrm{D}$ or $2 \mathrm{D}$ system. The mirror is tilted by two or more stack actors. Piezo devices with resonance frequencies up to $9 \mathrm{kHz}$ without masses for deflection in one direction (1D) are commercially available. The resonance frequency is lowered by the mirror masses. This is leading to resonance frequencies up to $6 \mathrm{kHz}$. It has to be mentioned that these systems are only useful at $80 \%$ of the resonance frequency. With two-dimensional tilting mirrors resonance frequencies of only $1 \mathrm{kHz}$ can be achieved without mirror masses. An increase of resonance frequency is possible by reducing mirror and actor masses. Micro-electro mechanical systems (MEMS) based on thin piezo-electrical layers are under investigation [10]. A disadvantage of piezo actuators is that the lifetime of these 
systems limited to $10^{10}$ cycles [12]. This corresponds to 555 operating hours at an oscillation frequency of $5 \mathrm{kHz}$. Fatigue in piezo crystal (e.g. micro cracks) and reduction of piezo-electrical characteristics of the crystal occur during operation time.

MEMS mirrors feature low masses thus leading to high resonance frequencies $(>20 \mathrm{kHz})$. The production of these systems is quite cheap because it is based on popular silicon technology. Very fine and thin structures can be fabricated by lithography, etching and metallization. Size of the mirror surface can be varied between some $100 \mu \mathrm{m}$ and several millimeters. The thickness of the mirror is in the range of $100 \mu \mathrm{m}$. Hanging of the mirror is done by hinges with widths $<30 \mu \mathrm{m}$. Two-dimensional beam deflection is possible with just one optical element by this setup. Deflection is generated by electromagnetic or electrostatic forces and in some cases by applied piezo layers. Electrostatic scanners are driven by four electrodes beneath the mirror. A voltage difference is applied to the mirror being the moving electrode and the electrodes below. It deflects based on the electrostatic force until a balance with spring forces is reached. These mirrors can be operated below and at resonance frequency at which maximum deflection angle and scan velocity occur. Angular range and resonance frequency are dependent on the stiffness of the hinges. Furthermore, the resonance frequency is influenced by size and mass of the mirror.

When using acousto-optical deflectors (AOD) an acoustic wave is coupled into a crystal generating a grating by changing the refractive index of the material. Time for varying the deflection angle is dependent on beam diameter and acoustic velocity in the material which is $4200 \mathrm{~m} \cdot \mathrm{s}^{-1}$ for the commonly used $\mathrm{TeO}_{2}$. Therefore, the variation of the deflection angle is in the MHz-range.

Table 1 Qualitative comparison of high dynamic beam deflection systems

\begin{tabular}{lcccc}
\hline & Size & $\begin{array}{c}\text { Geometric } \\
\text { flexibility }\end{array}$ & $\begin{array}{c}\text { Optical } \\
\text { elements }\end{array}$ & $\begin{array}{c}\text { Dy- } \\
\text { namic }\end{array}$ \\
\hline $\begin{array}{l}\text { Mechanical } \\
\text { scanner }\end{array}$ & - & - & 1 & + \\
$\begin{array}{l}\text { Galvanometric } \\
\text { scanner }\end{array}$ & -- & + & 2 & 0 \\
$\begin{array}{l}\text { Piezo scanner } \\
\text { (2D) }\end{array}$ & 0 & + & 1 & - \\
$\begin{array}{l}\text { MEMS scanner } \\
\begin{array}{l}\text { Acousto- } \\
\text { optical scanner }\end{array}\end{array}$ & ++ & + & 1 & + \\
\hline
\end{tabular}

A disadvantage of AODs is the not deflected beam of zeroth order which cannot be used in scanner systems. The diffraction efficiency is described by the distribution of the intensity between zeroth and first order. Commercially available deflectors have diffraction efficiencies in the range between 50 - $70 \%$. A maximum efficiency of $49 \%$ is possible for a two dimensional beam deflection if two acousto-optical deflectors are in series connection. Another problem for laser material processing is variation of the diffraction efficiency dependent on the deflection angle.
This is leading to a variation of the deflected beam which has to be adapted by temporal power modulation.

Due to the qualitative comparison MEMS mirrors reveal the highest potential regarding dimensions, geometric flexibility and dynamic behavior. Up to now, there is no experience in laser materials processing with laser powers of up to $100 \mathrm{~W}$ (cw) with such mirrors because these systems are being developed for use in display technology.

\section{Miniaturized scanner for high dynamic beam deflec- tion}

For first tests with MEMS scanners in laser material processing a miniaturized scanner is developed. A MEMS mirror is shown in Fig. 6. This scanner has a mirror diameter of $3 \mathrm{~mm}$; the resonance frequency is $940 \mathrm{~Hz}$ with a deflection angle of $35 \mathrm{mrad}$. The operation voltage is $400 \mathrm{~V}$. Because absorption of laser power is critical the reflectivity of the mirror has been measured between $500-2500 \mathrm{~nm}$ for a micro mirror with a gold coating as well as a dielectric enhanced coating (Fig. 6, right). In the range between 1000 - $1100 \mathrm{~nm}$ the dielectric enhanced mirror has a reflectivity of $99 \%$, the gold mirror of $96 \%$.
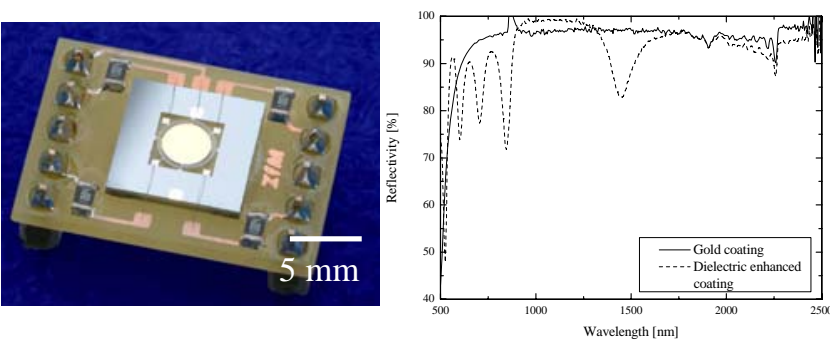

Fig. 6 MEMS mirror (left) and reflectivity over wavelength for gold coated and dielectric enhanced mirror (right)

For first functional tests a miniaturized scanner is designed, developed and built in which the beam deflection is done by a MEMS mirror. The exploded assembly drawing is shown in Fig. 7, left. The system consists of an incoupling unit for fiber laser collimator, a dichroitic mirror with a low reflectivity at $532 \mathrm{~nm}$, a fixture for the MEMS mirror and a focusing optics. The focusing optics is designed for telecentric imaging with a scan area of $2 \times 2 \mathrm{~mm}^{2}$ and a focus length of $50 \mathrm{~mm}$ and consists of three single lenses. Chromatic correction has been designed for the two wavelengths of $532 \mathrm{~nm}$ and $1070 \mathrm{~nm}$.
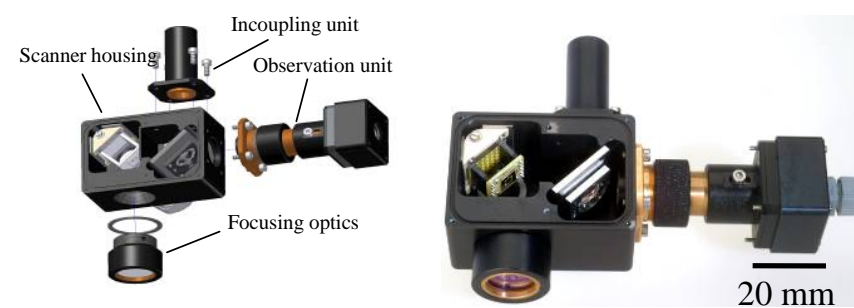

Fig. 7 Miniaturized scanner with MEMS mirror: 3D-CAD model (left) and prototypical realization (right)

By using a opto-mechanical tolerance analysis lens surfaces with the most important influence on optical aberrations are being identified. The mounting of the lenses has been designed with spacers; the first lens can be adjusted by compensators since this lens is very sensitive against 
deviations in $\mathrm{x}$ - and $\mathrm{y}$-direction. An observation unit is coaxially integrated into the optical path for beam positioning.

To reduce the size of the observation unit a Galilei telescope with a magnification of $5 x$ has been chosen. Due to the small aperture of the MEMS mirror $(3 \mathrm{~mm})$ the optical resolution is limited to $8.8 \mu \mathrm{m}$. Both telescope lenses are movable to realize a good image even out of focus. Prototypical realization of this system is shown in Fig. 7, right.

By thermographic imaging the temperature distribution of the irradiated MEMS mirror has been measured. For laser powers below $10 \mathrm{~W}$ no change in temperature is visible. In Fig. 8, left, the thermographic imaging at a laser power of $90 \mathrm{~W}$ is given. The mirror has a dielectric enhanced coating, the hinges are uncoated. Due to the coating no change in temperature can be detected for the mirror but due to the uncoated silicon the hinges are heated up to $120 \mathrm{~K}$. In the right picture of Fig. 8 the change in temperature in dependence of irradiation time (max. $3 \mathrm{~s}$ ) for two different laser powers $(60 \mathrm{~W}$ and $90 \mathrm{~W}$ ) is given.
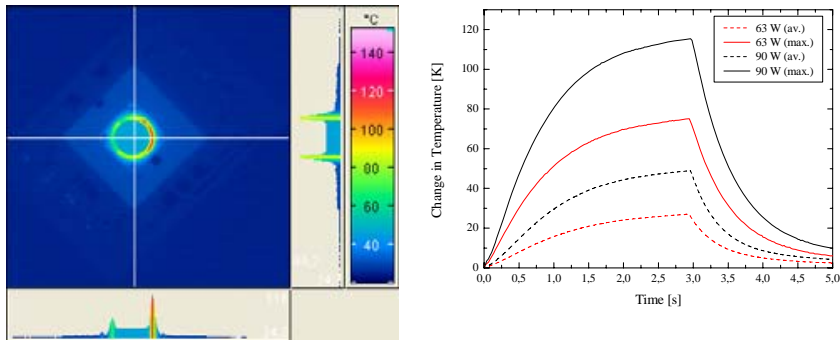

Fig. 8 Temperature distribution of irradiated MEMS mirror: thermographic imaging (left) and heating cycle (right)

Temperature measurements are performed in static operation without moving the micro mirror. Further examinations with moving mirror have to be carried out because an impact on the mechanical and dynamical characteristics of the MEMS mirror due to the thermal load is expected [13].

The intensity distribution is measured by using a CCD based diagnostics system. The Gaussian intensity distribution of the fiber laser is not influenced by the MEMS mirror or by the focusing optics. The focus diameter is about $0.2 \mathrm{~mm}$ while the collimated beam has a diameter of $0.8 \mathrm{~mm}$. To measure the dynamic behavior of the MEMS mirror this diagnostics system is used while oscillating the mirror. In the left picture of Fig. 9 the integration time of the device is set to $1030 \mu$ s what corresponds to an oscillation frequency of $930 \mathrm{~Hz}$.

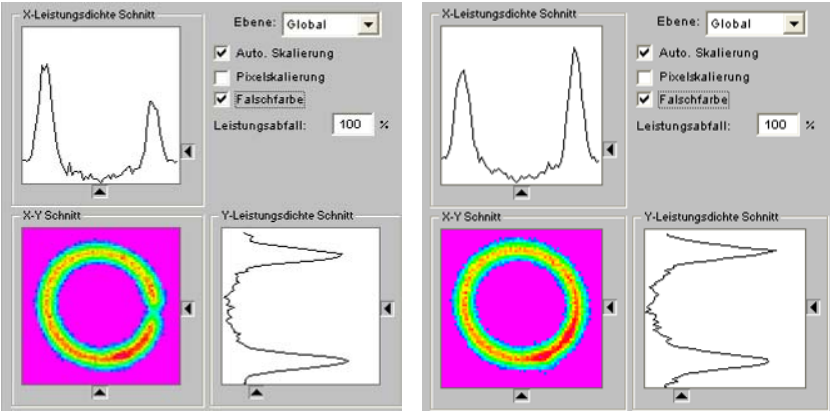

Fig. 9 Intensity distribution during oscillation: integration time for measurement $1030 \mu$ s (left), $1070 \mu$ s (right)

The ring shape is not closed until the integration time is raised to $1070 \mu$ s where one complete revolution of the laser focus is detected. The resulting oscillation frequency is $934 \mathrm{~Hz}$.

Further tests have to be carried out showing the possibility of MEMS scanner mirrors for laser beam materials processing with powers more than $10 \mathrm{~W}(\mathrm{cw})$. If these tests are successful changing of the mirror design is possible. Therefore, resonance frequencies are possible up to $20 \mathrm{kHz}$ at a mirror diameter of $3 \mathrm{~mm}$ by changing the stiffness of the micro hinges. At a resonance frequency of $1 \mathrm{kHz}$ mirror diameters between 5 - $10 \mathrm{~mm}$ are also possible.

\section{Laser beam micro welding with superposed oscilla- tion}

By superposing the described oscillation with linear feed the width and depth of the weld seems can be influenced precisely. Two cross sections of bead-on-plate welds performed with a galvanometer scanner in stainless steel are shown in Fig. 10.

In upper picture a weld carried out at a feed rate of $20 \mathrm{~mm} \cdot \mathrm{s}^{-1}$ with a modulation amplitude of $0.5 \mathrm{~mm}$ is shown. The cross section reveals a wide and homogenous melt zone with almost vertical edges. This leads to a uniform welding width over the complete welding depth. As a result the size of the supporting joint width in overlap joints is independent from welding depth.

For increased feed rate $\left(100 \mathrm{~mm} \cdot \mathrm{s}^{-1}\right)$ in combination with an amplitude of $0.5 \mathrm{~mm}$ solidification takes place within the single modulation cycles. Due to the high feed rate and high amplitude no homogeneous melt pool is build leading to intermittent solidification and remelting of the material. Different solidified welding tracks can be clearly distinguished in Fig. 10, as the overlap between the single modulation cycles is insufficient. 

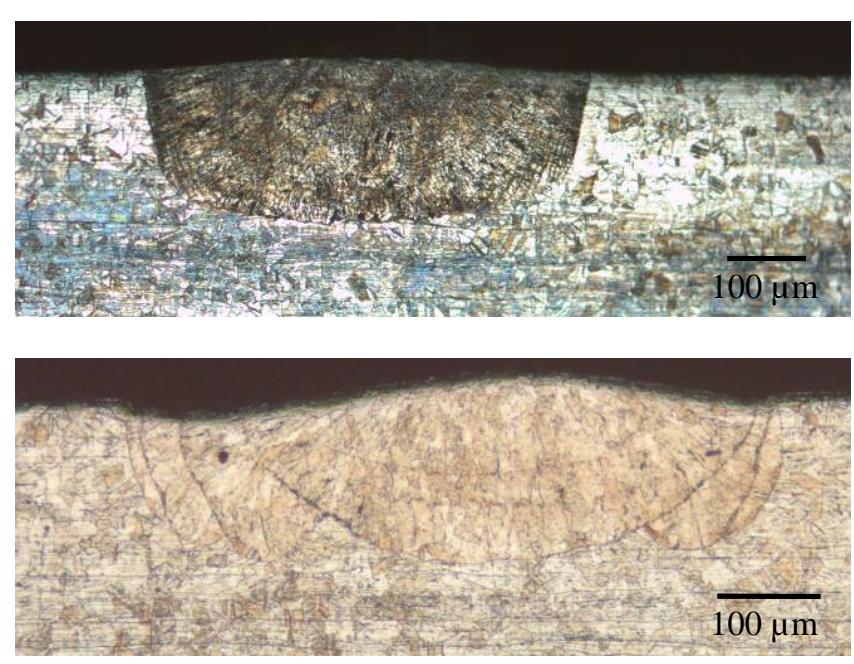

Fig. 10 Laser beam micro welding of stainless steel at different feed rates $[\mathrm{f}=1 \mathrm{kHz}, \mathrm{a}=0.5 \mathrm{~mm}$; up: $\mathrm{P}=100 \mathrm{~W}$, $\mathrm{v}=20 \mathrm{~mm} \cdot \mathrm{s}^{-1}$, below: $\mathrm{P}=200 \mathrm{~W}, \mathrm{v}=100 \mathrm{~mm} \cdot \mathrm{s}^{-1}$,]

The welding geometry is slightly asymmetric due to the asymmetric energy input as a result of the different energy input per length. The vectors of feed direction and superposed oscillation are in opposite directions for the right side of the welding seam and in the same direction for the left side resulting in a lower energy input.

Two cross sections of bead-on-plate welds in copper are shown in Fig. 11. At a laser power of $200 \mathrm{~W}$ and a feed rate of $30 \mathrm{~mm} \cdot \mathrm{s}^{-1}$ two different amplitudes $(0.1 \mathrm{~mm}$ in the left, $0.3 \mathrm{~mm}$ in the right picture) are chosen with an oscillation frequency of $1 \mathrm{kHz}$. Again the cross section of the weld seam is not v-shaped like a conventional weld seam but shows a u-shaped melting zone with almost vertical edges. At an amplitude of $0.1 \mathrm{~mm}$ the welding width is $340 \mu \mathrm{m}$ at a depth of $370 \mu \mathrm{m}$, for an amplitude of $0.3 \mathrm{~mm}$ the width increases to $0.5 \mathrm{~mm}$ with decreasing depth of $260 \mu \mathrm{m}$.
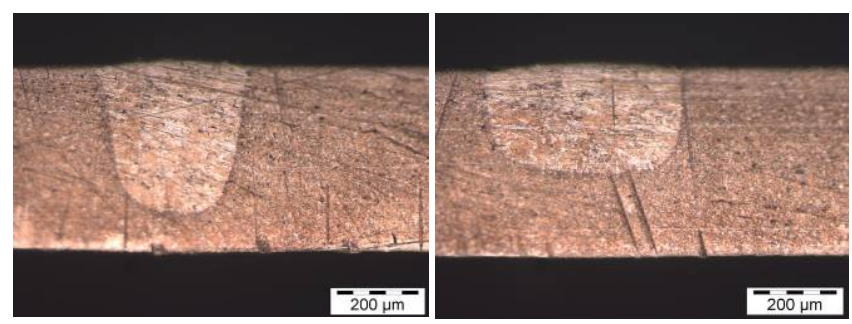

Fig. 11 Laser beam micro welding of copper (CuSn6) $\left[\mathrm{P}=200 \mathrm{~W}, \mathrm{v}=30 \mathrm{~mm} \cdot \mathrm{s}^{-1}, \mathrm{f}=1 \mathrm{kHz}, \mathrm{a}=0.1 \mathrm{~mm}\right.$ (left), $\mathrm{a}=0.3 \mathrm{~mm}$ (right)]

Thus, the welding geometry can be influenced by changing the preset amplitude. Therefore, it is an additional parameter for laser beam micro welding like feed rate and laser power whereby the oscillation frequency is not arbitrary because it depends on the feed rate and overlap. Furthermore a sufficient overlap of the single modulation cycles is a crucial quality criterion in order to ensure a homogenous melt pool with a high supporting joint width.

\section{Conclusion and outlook}

Superposed oscillation for laser beam micro welding shows a huge potential in stabilizing welding processes for difficult-to-weld materials and in adapting the welding geometry to the requirements, e.g. an unchanging welding width with increasing welding depth. Up to now, there are no high dynamic beam deflection systems available with oscillation frequencies $>20 \mathrm{kHz}$ as an alternative to commonly used galvanometric scanners. These systems are limited in their dynamic behavior to oscillation frequencies $<5 \mathrm{kHz}$. High oscillation frequencies are required for feed rates $>150 \mathrm{~mm} \cdot \mathrm{s}^{-1}$ to achieve a sufficient overlap which influences the joint quality significantly. A promising alternative are silicon-based MEMS mirrors with small dimensions and high dynamic. Unfortunately, these systems have been designed for display applications and there is no experience with high average powers. The examination of the applicability of these micro mirrors for laser material processing is subject to further investigations. For short-term irradiation and laser powers in the range of $100 \mathrm{~W}$ a change in temperature is measurable especially in the area of the micro hinges. The influence on the dynamic behavior of the micro mirror due to this heating has to be carried out in further examinations. Investigations on acousto-optical deflectors (AOD) are being conducted as an alternative deflection system. Due to oscillation frequencies of more than $100 \mathrm{kHz}$ just with these systems an influence on the behavior of the keyhole can be expected.

\section{Acknowledgments}

The authors gratefully acknowledge the financial support of the German Research Foundation (DFG) within the Collaborative Research Centre (SFB440) “Assembly of Hybrid Microsystems” at the Aachen University of Technology (RWTH Aachen).

\section{References}

[1] Y. Okamoto, A. Gillner, A. Olowinsky, J. Gedicke and Y. Uno: Fine micro-welding of thin stainless steel sheet by high speed laser scanning, Proc. of LPM 2007

[2] J. Gedicke, A. Olowinsky, J. Artal and A. Gillner: Influence of temporal and spatial laser power modulation on melt pool dynamics, Proc. of ICALEO 2007, Laser Materials Processing Conference, 816-822 (2007)

[3] A. Boglea, A. Olowinsky and A. Gillner: TWIST - A new method for the micro-welding of polymers with fibre lasers, Proc. of ICALEO 2007, Laser Materials Processing Conference, 136-142 (2007)

[4] K. Klages, J. Gedicke and A. Olowinsky: Pulse forming at laser beam micro welding, Proc. of ICALEO 2005, Laser Materials Processing Conference (2005)

[5] U. Dürr, R. Holtz and T. Westphäling: Materialspezifische Pulsleistungsmodulation beim Laserstrahl-Mikroschweißen; Proc. LEF 2003, 87-97 (2003)

[6] J. Wilden, S. Jahn et al.: Laserstrahlschweißen mit modulierbaren Nd:YAG Laserstrahlquellen - Wechselwirkungsmechanismen zwischen Leistungmodulation und Schmelzbadströmung sowie Erstarrung; DVSBerichte, 250, 425-429 (2008)

[7] A. Mahrle and E. Beyer: Control of the energy deposition during laser beam welding by oscillation techniques, Proc. of the $4^{\text {th }}$ Int. WLT-Conference on Lasers in Manufacturing, 97-103 (2007) 
[8] O. Berend, H. Haferkamp, O. Meier and L. Engelbrecht: High-frequency beam oscillating to increase the process stability during laser beam welding with high melt pool dynamics, Proc. of ICALEO 2005, Laser Materials Processing Conference, 1032-1041 (2005)

[9] M. G. Forrest and F. Lu: Advanced dual beam laser welding of zinc-coated steel sheets in lap joint configuration with zero gap at the interface, Proceedings ICALEO 2004, Laser Materials Processing Conference, (2004)

[10] W. Chen and P. Molian: Dual-beam laser welding of ultra-thin AA 5052-H19 aluminum, Advanced Manufacturing Technologies, 39, 6, 889-897 (2008)

[11] J.G. Smits, K. Fujimoto and V.F. Kleptsyn: Microeletromechanical flexure PZT actuated optical scanner: static and resonance behavior, Journal of Micromechanics and Microengineering, 15, 6, 1285-1293 (2005)

[12] F. Claeyssen, R. Le Letty et al.: Mechanisms based on piezo actuators, Proc. of the SPIE, 4332, 225-233 (2001)

[13] T. Kobayashi, T. Itoh, R. Sawada and R. Maeda: Tunable optical microscanner driven by piezoelectric actuator, Japanese Journal of Applied Physics, 46, 9, 6429-6432 (2007)

(Received: July 17, 2009, Accepted: June 23, 2010) 